\title{
An Unbiased Mineral Compositional Analysis Technique for Circumstellar Disks
}

\author{
Yung Kipreos* \& Inseok Song \\ Department of Physics \& Astronomy, University of Georgia, Athens, GA \\ https:// doi.org/10.33697/ ajur.2021.043 \\ Student:ykipreos@uga.edu* \\ Mentor:song@uga.edu
}

\begin{abstract}
A circumstellar disk that surrounds a star is composed of gas, dust, and rocky objects that are in orbit around it. Around infant stars, this disk can act as a source of material that can be used to form planetesimals, which can then accrete more material and form into planets. Studying the mineral composition of these disks can provide insight into the processes that created our solar system. The purpose of this paper is to analyze the mineral composition of these disks by using a newly created python package, Min-CaLM. This package determines the relative mineral abundance within a disk by using a linear regression technique called non-negative least square minimization. The circumstellar disks that are capable of undergoing compositional analysis must have a spectrum with both a detectable mid-infrared excess and prominent silicate features. From our sample, there are only eight debris disks that qualify to be candidates for the Min-CaLM program. The mineral compositions calculated by Min-CaLM are then compared to the Tholen asteroid classification scheme. HD 23514, HD 105234, HD 15407A, BD+20 307, HD 69830, and HD 172555 are found to have a compositions similar to that expected for C-type asteroids, TYC 9410-532-1 resembles the composition of S-type asteroids, and HD 100546 resembles D-type asteroids. Min-CaLM also calculates the mineral compositions of the comets Tempel 1 and Hale-Bopp, and they are used as a comparison between the material in our early solar system and the debris disk compositions.
\end{abstract}

\section{KEYWORDS}

Debris disk; Mineral; Composition; Analysis; Asteroid; Circumstellar; Spectroscopy; Python

\section{INTRODUCTION}

Circumstellar disks are rings of gas, dust, and other rocky objects that are in orbit around a star. Two types of common circumstellar disks are protoplanetary disks and debris disks. ${ }^{1,2}$ Protoplanetary disks form around infant stars before they reach the main sequence. The rocky material within these disks grows through violent collisions, eventually combining to form planetesimals. ${ }^{3}$ Debris disks form after the gas-rich protoplanetary disk around the star has dissipated, and they are composed primarily of dust grains and larger rocky bodies. ${ }^{4}$ The material in debris disks is composed of the remnants of collisions between planetesimals around the star. Several dust removal mechanisms eliminate dust grains effectively in the gas-poor debris disks in timescales much shorter than stellar ages, including: (1) Poynting-Robertson drag; (2) stellar wind drag; and (3) stellar radiation blowouts of small grains. ${ }^{5,6,7}$ The existence of debris disks indicates that this loss of material must be counterbalanced by the creation of new material via collisions of larger rocky material such as planetesimals. ${ }^{8}$ Therefore, the presence of a debris disk suggests the presence of planetesimals or already formed planets around the star. ${ }^{3}$

The light spectrum of debris disks can be used to determine physical characteristics such as the mineral composition, grain sizes, and temperature of the debris disk that emitted it. ${ }^{9}$ This paper will primarily study the mineral composition of debris disks. The mineral composition of a debris disk can be determined because the emitted spectrum is a combination of the individual spectra of the dust grains that compose the optically thin disk. Each dust grain mineral species produces a unique spectrum that allows the species to be identified. ${ }^{10}$ Thus, the mineral composition of a debris disk can be determined by deconstructing its emitted spectrum into the spectra of the individual mineral species within the disk.

Studying the mineral composition of debris disks in other solar systems may provide insights into the formation of our solar system. A particularly useful analog to the dust in other solar systems is the carbonaceous chondrites that are abundant in the asteroid belt in our solar system. ${ }^{11}$ Chondrite meteorites are thought to have been created from shock heating and thermal annealing in the protoplanetary disk of our early solar system. ${ }^{12}$ These thermal processes cause them to have a silicate-rich composition that is similar to the mineral composition detected in debris disks. ${ }^{13}$ This presents the possibility that the ongoing 
processes in the debris disks are the same processes that created our solar system. ${ }^{14}$ Therefore, the detection of debris disks with similar compositions to some constituents of the solar system provides a rare opportunity to study the evolution of our solar system.

The challenge of performing mineral spectroscopy on debris disks is the limited number of stellar candidates that meet the necessary qualifications for such analysis. Matthews et al. (2014) reported that debris disks are detected around $\sim 10 \%$ of solartype stars (e.g., the FEPS survey) and around $\sim 20 \%$ of FGK type stars (e.g., the DUNES survey). ${ }^{15,16}$ However, because of the detection sensitivity required to observe debris disks and the need for a highly sensitive space InfraRed telescope (e.g., Spitzer Space Telescope), the number of currently known stars that have debris disks is relatively small ( $\mathrm{N}=505$ from Cotten and Song 2016). ${ }^{17}$ The few stars that have debris disks must also have a detectable mid-infrared excess, prominent silicate mineral features, and have been observed by either the Infrared Space Observatory (ISO) or by the Spitzer Space Telescope (SST). ${ }^{5}$ For these reasons, out of 505 of the known debris disks, only eight are viable candidates for mineral composition analysis. ${ }^{17}$

Like chondrite meteorites, comets are composed of the material from the early solar system, primarily from the solar nebula during the protoplanetary disk phase. ${ }^{18}$ This cometary material is similar in composition to planetesimals found in systems with early planet formation. ${ }^{19}$ Therefore, studying the composition of comets is another way to study both the environment of the early solar system, and also the composition of distant planetesimals indirectly. ${ }^{20}$ To further study the cometary dust grains in our solar system, NASA conducted the Deep Impact study to measure the spectrum of the comet Tempel $1 .{ }^{21}$ In this study, an impactor was sent to collide with Tempel 1.22 The impact was necessary because the mineralogical emission features required for spectroscopy are only present when sufficiently small grains are abundant. ${ }^{3}$ The spectrum of Tempel 1 is representative of the composition of the early solar system when Tempel 1 is thought to have formed. ${ }^{23}$ One of the uses of the Deep Impact study is to compare the emission features of the early solar system dust against the emission features of debris disks. ${ }^{24}$ For this reason, Tempel 1 is an interesting candidate for mineralogical compositional analysis.

We developed a new mineralogical compositional analysis technique called Mineral Compositional Analysis using Least Square Minimization (Min-CaLM) and this technique was applied to the spectra of various comets and debris disks. We developed the Min-CaLM program to determine the mineral composition and relative abundance of each mineral within it. It should be noted that there are many possible combinations of minerals and relative mineral abundances that can produce the same observed spectrum of a certain target. ${ }^{11}$ Ordinarily, mineral compositional analysis is done manually, which could lead to potential bias in the results due to inherent biases the scientist may have. Unlike the traditional method, the Min-CaLM analysis provides a biasfree, purely mathematical composition result. However, we note that Min-CaLM results are not necessarily represent better fits. While it can only be applied to a few debris disks currently, our Min-CaLM method will be useful to analyze many more debris disk spectra that will be obtained with the Next Generation Space Telescope (James Webb Space Telescope).

It must be pointed out that, in this compositional analysis technique, we have made the underlying assumption that each debris disk is optically thin enough that the spectra of the inner disk can be measured. Min-CaLM studies the solid-state features in debris disk spectra, and these features originate in the optically thin region of the debris disks. By making this assumption, the composition calculated by Min-CaLM can be considered to be representative of the composition of the entire debris disk, not just the optically thin regions. This assumption allows the mineral spectra to be added together linearly to recreate the debris disk spectrum. Additionally, it should be noted that Min-CaLM does not consider the effects of different grain temperatures, shapes, or porosities in its calculations. These properties considerably affect the spectra produced by the dust particles, however, in MinCaLM's mineralogical library, each mineral is represented by a single spectrum with fixed temperature (Black-body), shape (circular), and porosity (minimal porosity).

\section{METHODS}

\section{The Debris Disk Selection Process}

The debris disks analyzed in this paper were chosen by first examining the list of 505 known debris disks from Cotten and Song (2016) which was the most complete list of nearby ( $<100 \mathrm{pc}$ ) debris disks $(>\sim 5 \mathrm{Myr})$ debris disks when published. The list did not contain proto-planetary disks because of the age selection criterion. The mid-IR spectra for some of these disks were taken by the IRS spectrometer onboard the Spitzer Space Telescope. ${ }^{25}$ These spectra were downloaded from the Spitzer Heritage Archive, which is a library that contains the data from past observations made by the Spitzer telescope. ${ }^{26}$ Of the 505 debris disks, Spitzer did not observe 98 of them, and therefore these disks could not be considered for mineral compositional analysis. In order to determine which of the remaining debris disks spectra have strong silicate mineral features, the spectral contributions of the stellar photospheres were subtracted from each of the Spitzer mid-IR debris disk spectra (Figure 1a). This subtraction separates the disk spectrum from the stellar photosphere spectrum. Additionally, large dust grains that compose the debris disk produce a smoothly varying blackbody emission that must be removed from the debris disk spectrum before it can undergo mineral spectroscopy. 
This approximation of blackbody emission from large dust grains is made so that the silicate spectral features of interest, produced by small dust grains, can be isolated and studied. When there exist strong hints of these dust blackbody contribution, we subtracted dust blackbody contribution with the best-fit blackbody dust temperature obtained by an iterative eye-fit.
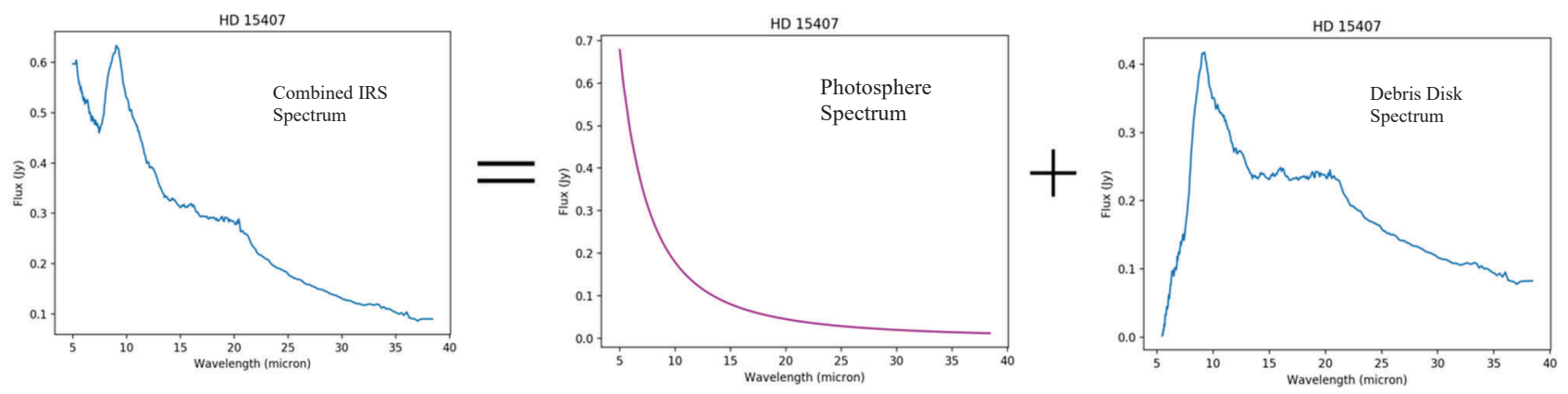

Figure 1. This figure demonstrates that the Spitzer IRS spectrum (left) is a combination of the spectral contributions from the stellar photosphere SED (Spectral Energy Distribution) (middle) and the debris disk spectrum (right). To obtain the isolated debris disk spectrum from the IRS spectrum, the stellar photosphere must be subtracted from the observed IRS spectrum.

Once the debris disk spectrum is isolated and the blackbody component has been removed, the presence of silicate emission features is determined. 397 of the 407 remaining debris disks do not have substantial silicate emission features (Figure 2), which leaves only eight candidates that are capable of undergoing mineral compositional analysis. Debris disk spectra with silicate features are rare because for this to be the case, there must be an abundance of small particles in the disk. Particles that are small enough to produce silicate features are easily blown out of the disk via radiation pressure. If a debris disk spectrum has prominent silicate features, then this implies that the small dust grains are being created faster than they are blown away from the disk. ${ }^{5}$ The debris disks that this paper will focus on are the disks around the following eight stars: BD+20 307; HD 172555; HD 15407A; HD 23514; HD 69830; TYC9410 532-1; HD 100546; and HD 105234.
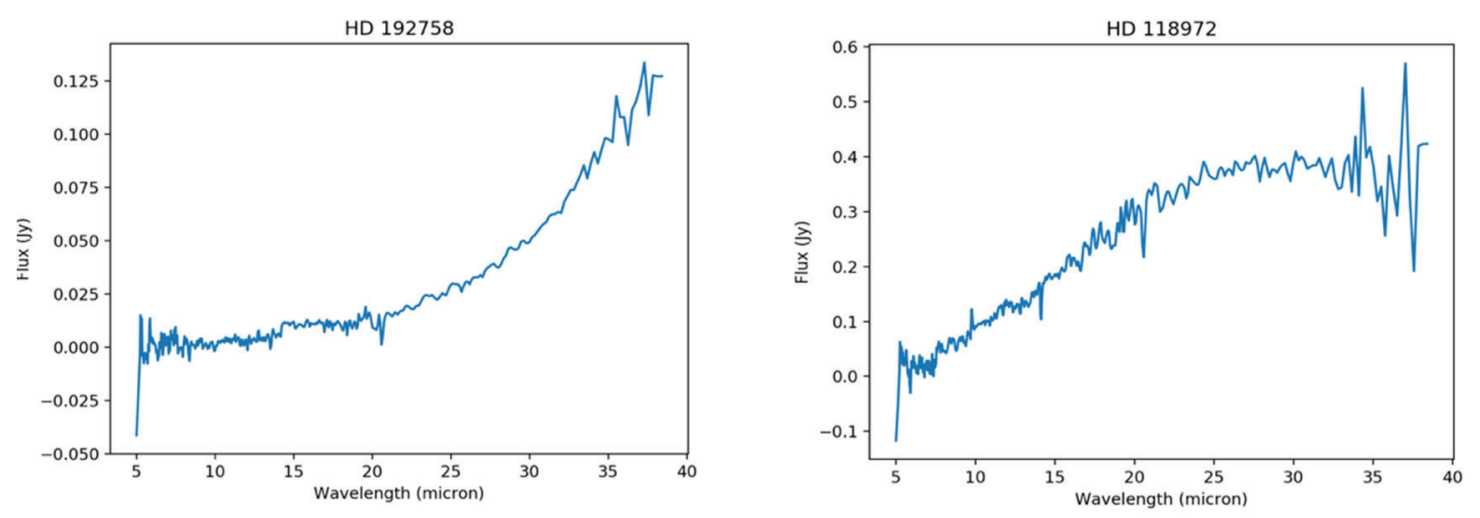

Figure 2. This figure displays two IRS spectra where silicate mineral features are not present. If these spectra contained silicate mineral features, the spectra would resemble the debris disk spectra in Figure 1.

In many of the debris disk spectra, there are sections of sharp decreases and increases in flux due to instrument artifacts (Figure 3). This issue originates from the reduction of the raw spectra obtained by the Infrared Spectrograph (IRS), an instrument in the Spitzer telescope. ${ }^{27}$ There are four spectral modules used in the IRS high resolution observing mode: Short-Low; Short-High; Long-Low; and Long-High. Each module covers a different range of infrared wavelengths. Each of these modules produces a spectrum with a flux scaling that is occasionally offset from the scaling produced by the other modules in the IRS. ${ }^{28}$ The spectral peaks from each module section are real features, but they appear to be artificially raised or lowered from the expected flux. One reason for the difference in flux levels is the different widths of the SL and LL slits in each module, which results in different amounts of flux lost. ${ }^{29}$ This issue is somewhat mitigated by using the CASSIS library which seeks to calibrate the scaling of each module. ${ }^{30}$ The CASSIS database contains optimally extracted spectra measured by each of the different Spitzer IRS modules, unlike the pipeline extracted spectra for general purpose from the Spitzer Heritage Database. ${ }^{31}$ For a small fraction of CASSIS reduced-IRS spectra, we see inappropriate inter-module scalings manifested as a sudden discontinuity of spectral shape at the module boundary. To correct the flux scaling for each instrument, we first plot the module spectra together. Misaligned sections 
of spectra are multiplied by a scaling factor, which is calculated by dividing the expected flux level by the actual observed flux at that wavelength. As an example, the result of this process on the circumstellar disk surrounding HD 15407A is displayed (Figure 3).
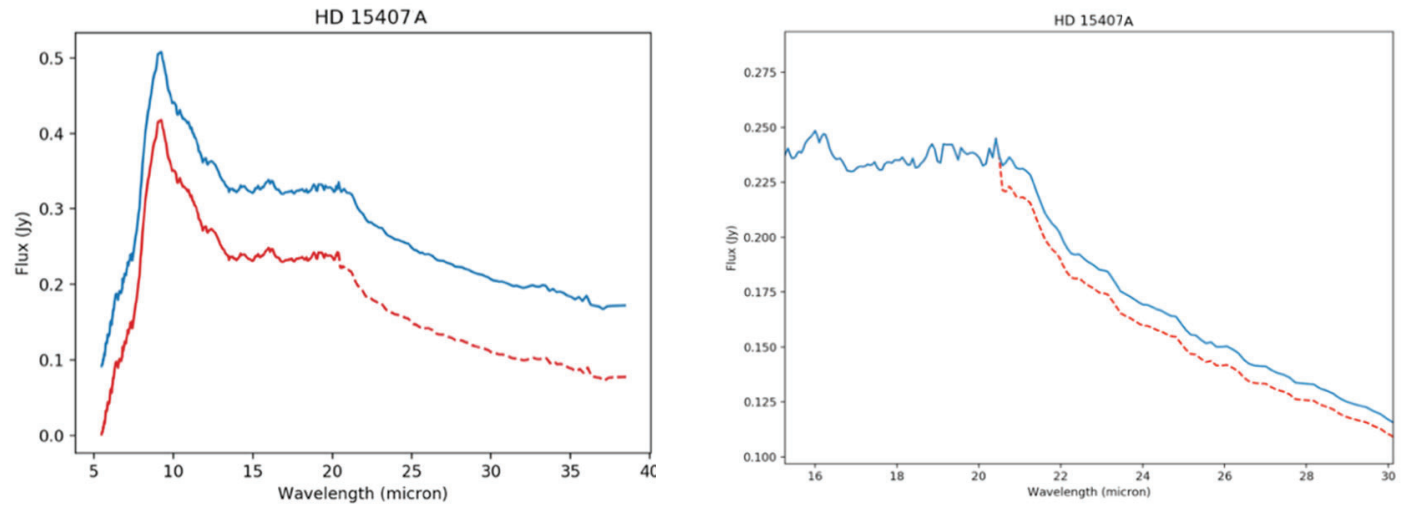

Figure 3. This figure compares the spectrum of the debris disk surrounding the star HD 15407A before (red) and after (blue) the spectrum was flux corrected. In the left figure, both spectra are displayed on top of each other to show the difference between them. The flux corrected spectrum (blue) is raised for demonstration purposes only. In the non-corrected spectrum (red), the flux scaling of the LL1 module (from 21 to 38 microns) is scaled too low compared to the rest of the module flux levels, so a sharp decrease can be seen around 21 microns. The right figure shows a close-up of the discontinuity, with both spectra plotted on top of each other for display purposes. The flux discontinuity can be seen at around $\sim 21$ microns. The flux-corrected spectrum should be continuous with no sharp discontinuities, so the LL1 module spectrum must be raised to the correct flux level compared to the other module levels.

\section{Traditional Methods of Mineralogical Analysis}

Traditionally, a mineral compositional analysis of debris disks has been performed manually. ${ }^{24}$ The minerals that are considered in the mineralogical analysis are chosen based on their likelihood of being present in a debris disk. ${ }^{23}$ The minerals are then selected based on the relative strength and wavelength of their spectral features and are then compared to the corresponding features in the debris disk spectrum. The mineral is considered to be a match if its spectral features have a similar height and wavelength to the features in the debris disk spectrum. The relative abundance of a mineral is the amount that its spectrum contributes to the overall debris disk spectrum. Therefore, the relative abundance is related to the amount of that mineral that is present in the disk. ${ }^{32}$ The debris disk spectrum is recreated by multiplying each mineral by its relative abundance within the disk and then adding the weighted mineral spectra together. If the minerals and relative abundances are well-chosen, then the newly created spectrum will resemble the original debris disk spectrum.

\section{The Min-CaLM Algorithm}

The manually driven phase space search method relies on a human observer to choose a small number of expected mineral species and guide the phase space search by hand, and thus is potentially limited by the initial choice of minerals and bias of the observer and their ability to search phase space. In order to improve this, we began developing the Min-CaLM program to mineralogically fit the IRS spectra of the debris disks around two stars initially, HD 23514 and HD 15407A, and modified the program until it was able to recreate both spectra successfully. The process of this development involved trying different mathematical methods to determine the relative abundance of each mineral to produce the best spectrum fit for both debris disks. The most effective mathematical approach was found to be a linear regression technique called non-negative least square minimization (NNLS). ${ }^{33}$ NNLS is a good method for compositional mineral analysis because the target spectrum (debris disk or comet), mineral spectra, and the relative abundances of each mineral create a linear system (Figure 4). Additionally, this is an overdetermined system, meaning that there are more equations than there are unknowns. This indicates that the solution to this linear system (i.e., the relative abundances of each mineral) is not unique, and therefore different combinations of mineral abundances could be used to create the same spectrum. NNLS provides a good approximation of the solution of an overdetermined system. ${ }^{34}$

To be able to use NNLS on the debris disk system, first, the target spectrum, mineral spectra, and relative abundances must all be converted into matrix form (Figure 4). The mineral spectra matrix is created by placing each mineral spectrum horizontally next to each other. This is a $903 \times 40$ size matrix because there are 40 minerals in the Min-CaLM mineral library, and there are 903 spectral points in each mineral spectrum. Likewise, the data points of the debris disk spectrum are resampled into a $903 \times 1$ matrix to match the number of spectral points of mineral spectra, and the relative abundances are stored in a $40 \times 1$ matrix because each mineral has only one abundance. This system of matrices forms a linear system, as shown in Figure 4 . In this matrix 
representation, $\left[t_{1}, t_{2}, t_{3}, \ldots, t_{903}\right]$ represents an IRS spectrum of a debris disk, while $\left[m_{1, i}, m_{2, i}, m_{3, i}, \ldots, m_{903, i}\right]$ represents a mineral spectrum of the $i^{t h}$ species in the mineral spectral library, and $\left[w_{1}, w_{2}, w_{3}, \ldots, w_{40}\right]$ corresponds to the best-fit relative abundances of all mineral species considered.

\begin{tabular}{|c|c|c|c|c|c|c|}
\hline$m_{1,1}$ & $m_{1,2}$ & $m_{1,3}$ & $\cdots$ & $m_{1,40}$ & $w_{1}$ & \\
\hline$m_{2,1}$ & $m_{2,2}$ & $m_{2,3}$ & $\ldots$ & $m_{2,40}$ & $w_{2}$ & \\
\hline$m_{3,1}$ & $m_{3,2}$ & $m_{3,3}$ & $\ldots$ & $m_{3,40}$ & $w_{3}$ & $=$ \\
\hline$\vdots$ & $\vdots$ & $\vdots$ & $\ddots$ & $\vdots$ & : & \\
\hline$m_{903,1}$ & $m_{903,2}$ & $m_{903,3}$ & $\cdots$ & $m_{903,40}$ & $w_{40}$ & \\
\hline
\end{tabular}

Figure 4. This is the matrix representation of the linear system containing the mineral spectra (left), the relative abundances of those minerals (middle), and the debris disk spectrum (right). The mineral spectra matrix is of size $903 \times 40$. Each column of this matrix corresponds to the individual spectrum of a mineral which contains 903 data points. The relative abundance matrix is of size $40 \times 1$ because each mineral has one relative abundance and there are 40 total minerals. The debris disk spectrum of size $903 \times 1$ is created by multiplying the mineral spectra and the relative abundance matrices.

Now that each part of the system is in matrix form, the NNLS equation (1) can be applied,

$$
\operatorname{argmin}|| \mathrm{mw}-\mathrm{t} \| \text { with } w_{i} \geq 0
$$

Here $\mathbf{m}$ represents the mineral spectra matrix, $\mathbf{t}$ is the target spectrum, and $\mathbf{w}$ is the relative abundance of each mineral.

Additionally, relative abundances are constrained to be zero or positive numbers. The reason for this constraint is that a negative mineral abundance would be non-physical. The result of the solved NNLS equation is a list of 40 mineral abundances. If a mineral is determined by Min-CaLM not to be present in the debris disk, then the abundance of that mineral will be zero. The new debris disk spectrum is recreated by multiplying the spectrum of each mineral with its corresponding relative abundance within the disk. Then each of the weighted spectra is added together. We list all 40 mineral species considered in our Min-CaLM scheme in Table 1.

\section{RESULTS}

The goal of this study is to calculate the unbiased mineral composition of eight debris disks and two comets using our newly developed mineralogical analysis technique, Min-CaLM. Before using the Min-CaLM scheme, each target spectrum must undergo the photosphere and blackbody removal processes described in the Methods section. The relative mineral abundances calculated by Min-CaLM are displayed in Table 2 for each target object. These abundances are used to recreate the spectrum of each object, shown in Figure 5. Also included in Table 2 are the black body temperatures used in Min-CaLM's calculations and reduced chisquared test results for each object. For illustrative purposes, a diagram of the mineral spectra present in the circumstellar disk of BD+20 307 is included in Figure 7 to demonstrate how the mineral spectra are combined to recreate the observed spectrum.

The reduced chi-square statistic is defined as the chi-square per degree of freedom and is used to determine the goodness of fit of the Min-CaLM recreated and the original spectra. For the reduced chi-square statistic, a fit is considered to be bad if $\chi_{v}^{2}>1$, good if $\chi_{v}^{2}<1$, and over-fitted if $\chi_{v}^{2}<<1 .{ }^{35}$ For the purposes of Min-CaLM, an overfitted result is acceptable because the objective of the program is to reproduce the target spectrum as closely as possible. Of the results calculated by Min-CaLM, HD 172555, BD+20 307, HD 100546, and Hale Bopp have chi square results less than one, and HD 15407A, HD 105234, HD 23514, HD 69830, TYC 9410-532-1, and Tempel 1 have chi square results larger than one. It should be noted that any model that produces an acceptable chi-square values should be treated as valid. This system is over-determined, so there are many possible solutions, and if those solutions produce acceptable chi-square results, then they are just as valid as these solutions.

The chi-square test values are calculated with $\left(o b s_{i}-f i t_{i}\right)^{2} /$ noise $_{i}^{2}$, which is then divided by the degrees of freedom, equal to the number of data points, and where $i$ indicates a wavelength point and the noise is the error recorded by Spitzer. Chi-square values are also plotted against the wavelength for each target object in Figure 6. Some diagrams in Figure $\mathbf{6}$ have peaks with high chi-square values, but the overall reduced chi-square value is small. This could indicate that the fitting is good at most wavelengths except for the wavelengths with high peaks. Together, these plots illustrate which wavelengths Min-CaLM struggles the most to fit. The chi-square value vs wavelength plot of an optimally reproduced target spectrum would hover around zero, and regions with a raised chi-square value indicate where the Min-CaLM-reproduced spectrum did not correctly reproduce the target 
spectrum. For instance, in the plot of HD 100546 in Figure 6, Min-CaLM struggled to fit the debris disk spectrum of HD 100546 around 6 and 11 microns, with the largest peaks in the plot at 6.35 microns. This plot indicates that Min-CaLM is possibly missing the species of phyllosilicates and olivine minerals that produce spectral peaks around those wavelengths. ${ }^{36}$ As evidenced from the plots in Figure 6, the mineralogical library of Min-CaLM is not yet complete and would considerably benefit from the inclusion of more olivine, silicate, and phyllosilicate mineral species.

\begin{tabular}{|c|c|c|c|}
\hline Mineral Name & Mineral Family & Formula & Description \\
\hline Tridymite & Tectosilicate & $\mathrm{SiO}_{2}$ & A tectosilicate mineral found in felsic igneous rocks \\
\hline Albite & Tectosilicate & $\mathrm{NaAlSi}_{3} \mathrm{O}_{8}$ & A feldspar mineral found in felsic igneous rocks \\
\hline Anorthite & Tectosilicate & $\mathrm{NaAlSi}_{3} \mathrm{O}_{8}$ & A feldspar mineral found in mafic igneous rocks \\
\hline Hypersthene & Inosilicate & $(\mathrm{Mg}, \mathrm{Fe}) \mathrm{SiO}_{3}$ & $\begin{array}{l}\text { An inosilicate mineral found in igneous rocks and } \\
\text { occasionally in metamorphic rocks }\end{array}$ \\
\hline Diopside & Inosilicate & $\mathrm{CaMgSi}_{2} \mathrm{O}_{6}$ & A silicate mineral found in ultramafic igneous rocks \\
\hline Hornblende & Inosilicate & $\mathrm{Ca}(\mathrm{Mg}, \mathrm{Fe})_{4} \mathrm{Al}\left(\mathrm{Si}_{7} \mathrm{Al}\right) \mathrm{O}_{22}(\mathrm{OH}, \mathrm{F})_{2}$ & $\begin{array}{l}\text { An inosilicate mineral found in metamorphic and igneous } \\
\text { rocks }\end{array}$ \\
\hline Wollastonite & Inosilicate & $\mathrm{CaSiO}_{3}$ & A calcium inosilicate mineral \\
\hline Nontronite & Phyllosilicate & $\begin{array}{l}\mathrm{Ca} 0.5\left(\mathrm{Si}_{7} \mathrm{Al}_{0.8} \mathrm{Fe}_{0.2}\right) \\
\left(\mathrm{Fe}_{3.5} \mathrm{Al}_{0.4 \mathrm{Mg}} \mathrm{Mg}_{0.1}\right) \mathrm{O}_{20}(\mathrm{OH})_{4}\end{array}$ & An iron-rich clay mineral found in basalt rocks \\
\hline Montmorillonite & Phyllosilicate & $(\mathrm{Na}, \mathrm{Ca})_{3}(\mathrm{Al}, \mathrm{Mg})_{2} \mathrm{Si}_{4} \mathrm{O}_{10}(\mathrm{OH})_{2}\left(\mathrm{H}_{2} \mathrm{O}\right)$ & A member of the smectite mineral group related to clay \\
\hline Saponite & Phyllosilicate & $\begin{array}{l}\mathrm{Ca}_{0.25}(\mathrm{Mg}, \mathrm{Fe})_{3} \\
\left((\mathrm{Si}, \mathrm{Al})_{4} \mathrm{O}_{10}\right)(\mathrm{OH})\left(\mathrm{H}_{2} \mathrm{O}\right)\end{array}$ & A smectite group mineral found in basalt rocks \\
\hline Halloysite & Phyllosilicate & $\mathrm{Al}_{2} \mathrm{Si}_{2} \mathrm{O}_{5}(\mathrm{OH})_{4}$ & A clay mineral sometimes found in basalt rocks \\
\hline Talc & $\begin{array}{l}\text { Hydrated } \\
\text { Magnesium Silicate }\end{array}$ & $\mathrm{Mg}_{3} \mathrm{Si}_{4} \mathrm{O}_{10}(\mathrm{OH})_{2}$ & A clay mineral commonly found in metamorphic rocks \\
\hline Olivine & $\begin{array}{l}\text { Nesosilicate } \\
\text { (Olivine group) }\end{array}$ & $(\mathrm{Mg}, \mathrm{Fe})_{2} \mathrm{SiO}_{4}$ & $\begin{array}{l}\text { A magnesium iron silicate commonly found in the upper } \\
\text { mantle }\end{array}$ \\
\hline Forsterite & $\begin{array}{l}\text { Nesosilicate } \\
\text { (Olivine group) }\end{array}$ & $\left(\mathrm{Mg}_{2} \mathrm{SiO}_{4}\right)$ & $\begin{array}{l}\text { A magnesium-rich olivine mineral commonly found in } \\
\text { igneous rocks, metamorphic rocks, and meteorites }\end{array}$ \\
\hline Fayalite & $\begin{array}{l}\text { Nesosilicate } \\
\text { (Olivine group) }\end{array}$ & $\mathrm{Fe}_{2} \mathrm{SiO}_{4}$ & $\begin{array}{l}\text { An iron-rich olivine mineral commonly found in alkaline } \\
\text { igneous (specifically volcanic) }\end{array}$ \\
\hline Bronzite & Pyroxene & $(\mathrm{Mg}, \mathrm{Fe}) \mathrm{SiO}_{3}$ & Magnesium silicate with containing amounts of iron \\
\hline Hedenbergite & Pyroxene & $\mathrm{CaFeSi}_{2} \mathrm{O}_{6}$ & An iron-rich pyroxene mineral found in chondrites \\
\hline Diopside & $\begin{array}{l}\text { Inosilicate } \\
\text { (Pyroxene group) }\end{array}$ & $\mathrm{MgCaSi}_{2} \mathrm{O}_{6}$ & $\begin{array}{l}\text { A monoclinic pyroxene mineral commonly found in igneous } \\
\text { and mafic rocks }\end{array}$ \\
\hline Enstatite & $\begin{array}{l}\text { Inosilicate } \\
\text { (Pyroxene group) }\end{array}$ & $\mathrm{MgSiO}_{3}$ & $\begin{array}{l}\text { A pyroxene silicate mineral commonly found in igneous and } \\
\text { metamorphic rocks }\end{array}$ \\
\hline Magnesite & Carbonate & $\mathrm{MgCO}_{3}$ & A carbonate mineral that contains traces of iron and nickel \\
\hline Dolomite & Carbonate & $\mathrm{CaMg}\left(\mathrm{CO}_{3}\right)_{2}$ & An anhydrous carbonate mineral \\
\hline Siderite & Carbonate & $\mathrm{FeCO}_{3}$ & A mineral composed of $\sim 48 \%$ iron \\
\hline Corundum & Oxide & $\mathrm{Al}_{2} \mathrm{O}_{3}$ & A translucent crystalline form of aluminum oxide \\
\hline Ti3O5 † & Oxide & $\mathrm{Ti}_{3} \mathrm{O}_{5}$ & An oxide mineral \\
\hline Hematite & Oxide & $\mathrm{Fe}_{2} \mathrm{O}_{3}$ & A gray colored iron ore \\
\hline Magnetite & Oxide & $\mathrm{Fe}_{2} \mathrm{O}_{4}$ & A magnetic iron ore \\
\hline $\mathrm{SiO} *$ & Other & $\mathrm{SiO}$ & Silicon monoxide gas \\
\hline Quartz & Oxide & $\mathrm{SiO}_{2}$ & A mineral commonly found in felsic igneous rocks \\
\hline Gamma Alumina † & Oxide & $\mathrm{Al}_{2} \mathrm{O}_{3}$ & A chemical compound related to corundum \\
\hline
\end{tabular}

Table 1. This table displays the minerals included in the Min-CaLM mineralogical library. Beside each mineral is the mineral group, the chemical formula, and a brief description of the mineral. The mineral group is a collection of minerals based on their chemical compositions. Min-CaLM calculated the emission for 19 of these minerals, but found no traces of them in the debris disk spectra, so those minerals are not displayed in this table. A $†$ symbol means that the mineral spectra was downloaded from the USGS Spectral Library, a * symbol indicates that the spectrum was obtained by tracing a spectral graph, and no symbol means that the mineral spectrum was downloaded from the ASU Spectral Library. The ASU Spectral Library contains mineral absorption spectra, but the Min-CaLM program requires the emission spectrum of each mineral. To convert the mineral absorption spectra to emission spectra, each spectrum is inverted along the y-axis (flux) via the equation [emission flux $=1-$ absorption flux] at each wavelength. 
The mineral composition of the debris disks is significant because they bear a resemblance to the composition of the asteroids in our solar system. Based on the Tholen Taxonomy classification of asteroids, the compositions of the debris disks calculated by Min-CaLM are similar to the composition of three of the most abundant asteroid types in our solar system. Six debris disks (HD 23514, HD 105234, HD 15407A, BD+20 307, HD 69830, and HD 172555) are found to have compositions similar to that of Ctype asteroids, one debris disk (TYC 9410-532-1) resembles the composition of S-type asteroids, and one debris disk (HD 100546) resembles D-type asteroids. These findings will be discussed in further detail in the Discussion section.

\begin{tabular}{|c|c|c|c|c|c|c|c|c|c|c|}
\hline \multirow[t]{2}{*}{ Mineral Name } & \multicolumn{10}{|c|}{ Targets } \\
\hline & $\begin{array}{l}\text { HD } \\
15407 \mathrm{~A}\end{array}$ & $\begin{array}{l}\text { HD } \\
172555\end{array}$ & $\begin{array}{l}\text { HD } \\
105234\end{array}$ & $\begin{array}{l}\mathrm{BD}+20 \\
307\end{array}$ & $\begin{array}{l}\text { HD } \\
23514\end{array}$ & $\begin{array}{l}\text { HD } \\
100546\end{array}$ & $\begin{array}{l}\text { HD } \\
69830\end{array}$ & $\begin{array}{l}\text { TYC } \\
9410 \\
532-1\end{array}$ & Tempel 1 & $\begin{array}{l}\text { Hale } \\
\text { Bopp }\end{array}$ \\
\hline $\begin{array}{l}\text { Blackbody } \\
\text { Temperature (K) }\end{array}$ & 344 & 373 & 425 & 424 & 372 & 336 & 330 & 360 & 485 & - \\
\hline Chi-square test result & 8.75 & 0.268 & 15.6 & 0.00610 & 59.0 & 0.0263 & 126 & 11.0 & 606 & 0.0238 \\
\hline Magnetite & 15.1 & 18.9 & - & - & 21.7 & - & 23.6 & 14.8 & 24.4 & - \\
\hline Hornblende & 15.0 & 26.9 & - & - & 9.8 & 69.0 & 45.3 & 12.1 & 5.5 & 47.1 \\
\hline Fayalite & - & 20.1 & - & - & - & 16.1 & 3.5 & - & 2.8 & - \\
\hline Forsterite & - & - & 6.9 & 8.4 & 7.4 & - & - & 10.1 & 10.1 & - \\
\hline Siderite & 0.6 & - & 3.0 & - & 2.6 & - & - & 1.1 & 3.2 & - \\
\hline Olivine & 13.9 & 15.1 & - & 8.7 & - & 7.6 & 18.3 & - & 5.8 & 24.4 \\
\hline Quartz & - & 6.2 & - & - & 6.2 & - & - & 3.7 & 0.9 & 1.2 \\
\hline Tridymite & 5.1 & - & - & - & 19.1 & - & - & 0.6 & 4.2 & - \\
\hline Hypersthene & - & - & 1.0 & 33.3 & 6.3 & - & - & - & - & - \\
\hline Magnesite & - & - & - & - & - & 7.3 & 0.4 & 1.8 & 3.2 & - \\
\hline Enstatite & - & - & - & - & - & - & - & - & 5.7 & 11.8 \\
\hline Diopside & - & - & - & 3.1 & 1.4 & - & - & 5.4 & - & - \\
\hline Dolomite & - & - & - & - & - & - & - & 9.6 & - & - \\
\hline Saponite & - & - & 49.4 & - & - & - & 3.8 & - & - & - \\
\hline Talc & - & - & - & 18.6 & 1.7 & - & - & 8.3 & 5.0 & 1.8 \\
\hline $\mathrm{Al} 2 \mathrm{O} 3$ & 6.3 & - & 5.9 & 7.6 & 1.0 & - & 0.2 & 8.2 & 2.4 & 10.7 \\
\hline Nontronite & - & 3.9 & - & - & - & - & - & - & - & - \\
\hline Bronzite & 8.1 & - & - & - & 1.6 & - & - & - & - & - \\
\hline $\mathrm{SiO}$ & - & - & 12.9 & - & - & - & - & - & - & - \\
\hline Corundum & 13.1 & - & - & - & 8.0 & - & 5.0 & 13.4 & 6.9 & 2.9 \\
\hline Halloysite & 5.6 & - & - & 7.0 & 5.2 & - & - & 2.8 & - & - \\
\hline Albite & 2.6 & - & - & 2.8 & 4.4 & - & - & 5.0 & 5.5 & - \\
\hline Wollastonite & - & - & - & - & 1.0 & - & - & - & - & - \\
\hline Anorthite & 2.2 & - & 1.1 & 0.6 & - & - & - & 3.3 & 4.8 & - \\
\hline Gamma Alumina & 3.6 & - & 1.0 & 10.0 & 2.7 & - & - & - & - & - \\
\hline Hematite & - & 9.0 & 0.8 & - & - & - & - & - & - & - \\
\hline Ti3O5 & - & - & 15.0 & - & - & - & - & - & 6.2 & - \\
\hline Montmorillonite & - & - & 3.1 & - & - & - & - & - & - & - \\
\hline Hedenbergite & - & - & - & - & - & - & - & - & 3.60 & - \\
\hline
\end{tabular}

Table 2. The relative \% abundance of each target spectrum, calculated by the Min-CaLM analysis. There are eight debris disks and two comets. Each target is labeled at the top of the table and the minerals are on the left-hand side. If a mineral is not calculated to be in the target spectrum this is denoted by a "_" symbol. The number in each cell represents the percentage of that mineral calculated to be present in the target spectrum. The spectrum of Hale Bopp does not show a hint of smooth blackbody continuum hence no blackbody temperature in the table. 

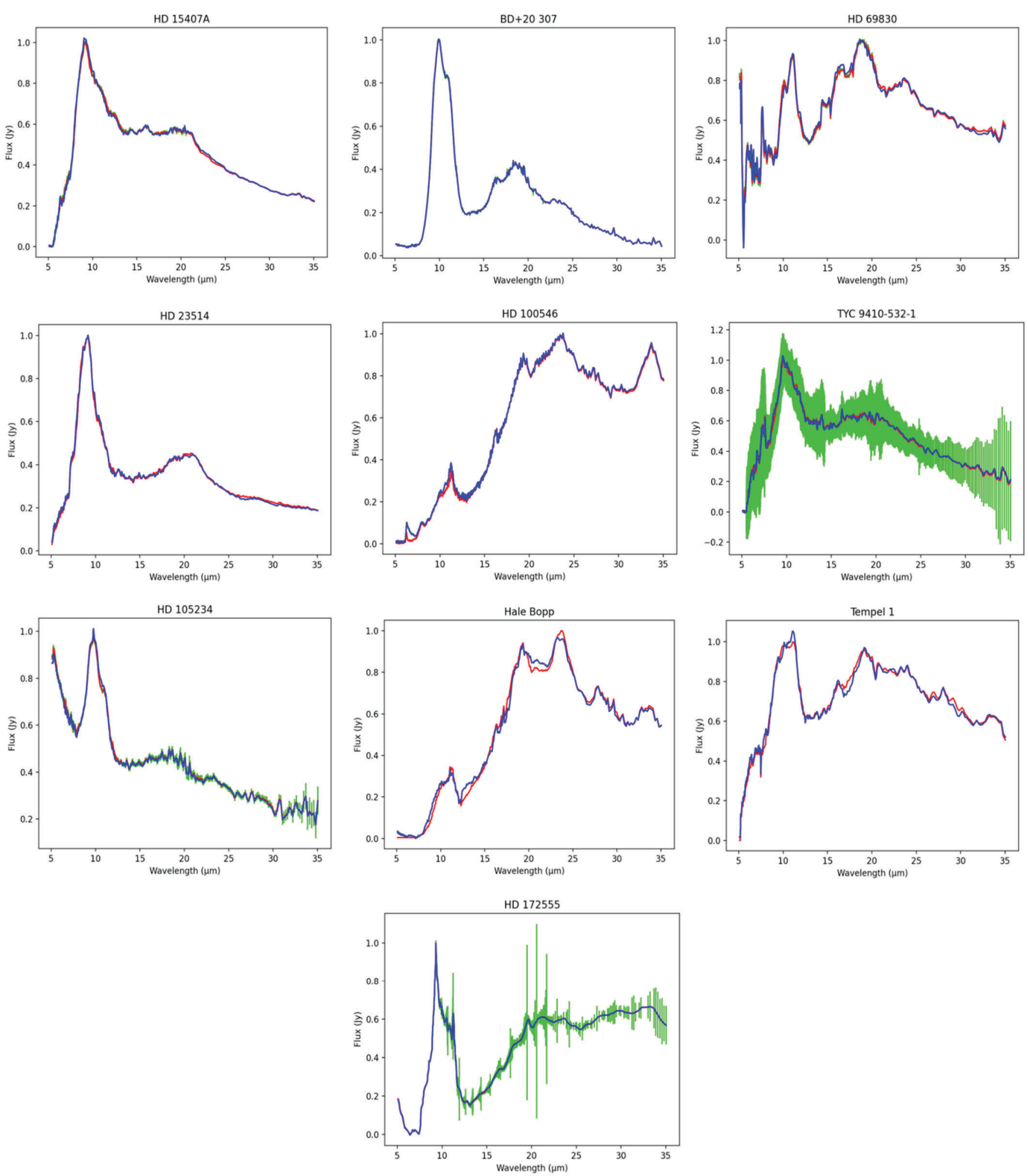

Figure 5. This figure displays the final recreated spectra of the Min-CaLM program. The y-axis of each graph is the flux measured in Jy, and the $\mathrm{x}$-axis is the wavelength measured in $\mu \mathrm{m}$. The red spectrum is the original photosphere-subtracted disk spectrum of a target object, and the blue spectrum is the recreated best-fit spectrum from Min-CaLM plotted on top of it. The green uncertainty bars represent the uncertainty in the observations made by the Spitzer telescope. It should be noted that in some diagrams, such as the diagram of HD 23514 the Spitzer uncertainty bars from the CASSIS library are too small to be visible. Hale Bopp and Tempel 1 were not observed by Spitzer, and 

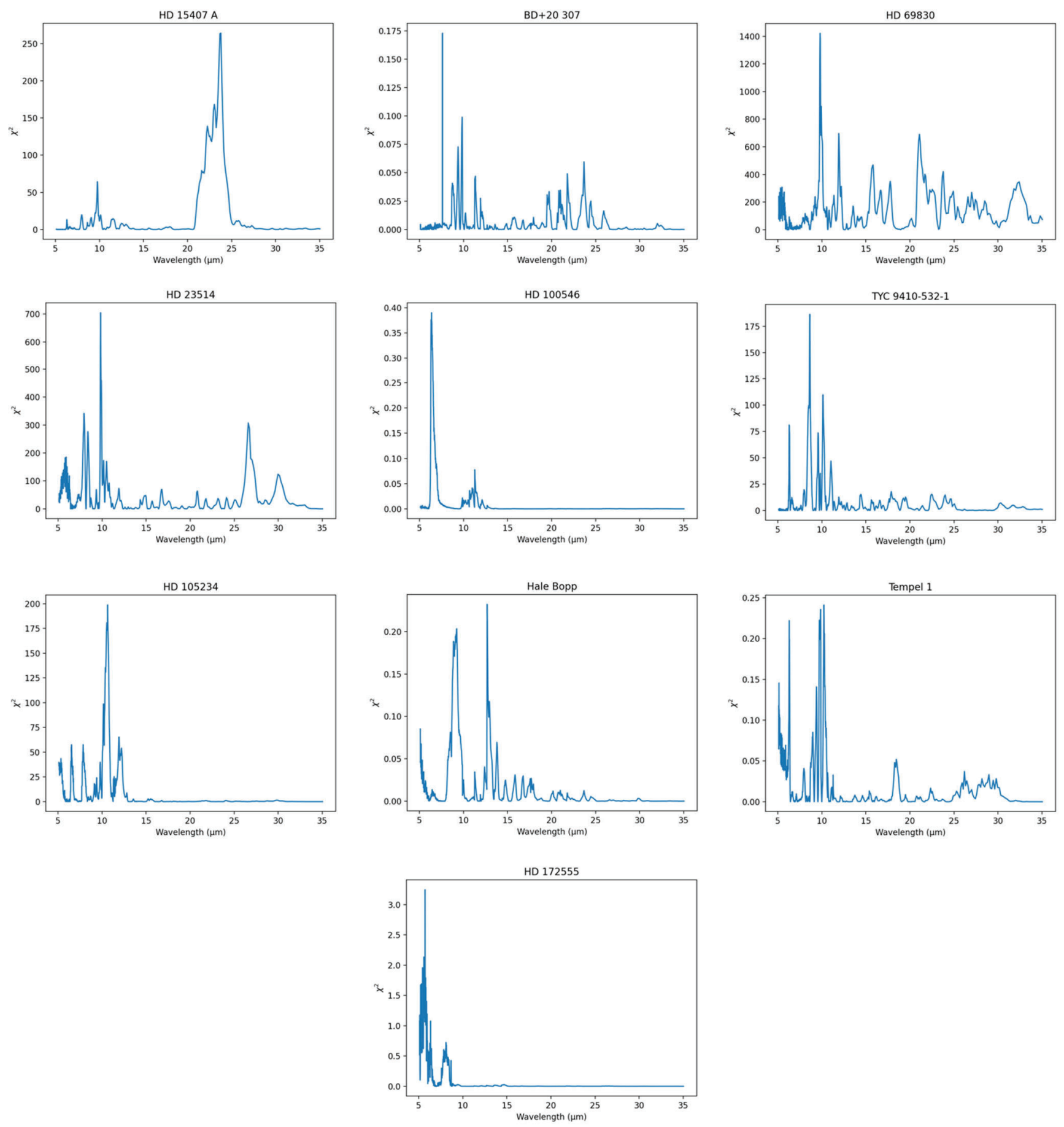

Figure 6. This figure displays the chi-square value vs wavelength for each Min-CaLM calculated spectrum compared to the original measured spectrum. The chi-square value vs wavelength plot for a perfectly matched reproduced spectrum should hover around zero. Therefore, the regions of wavelength that correspond to large chi-square values indicate where Min-CaLM has struggled to properly reproduce the target spectrum. These wavelength regions are thought to have not been properly reproduced because Min-CaLM's mineralogical library is missing the ideal corresponding mineral that contains peaks at that wavelength region. 


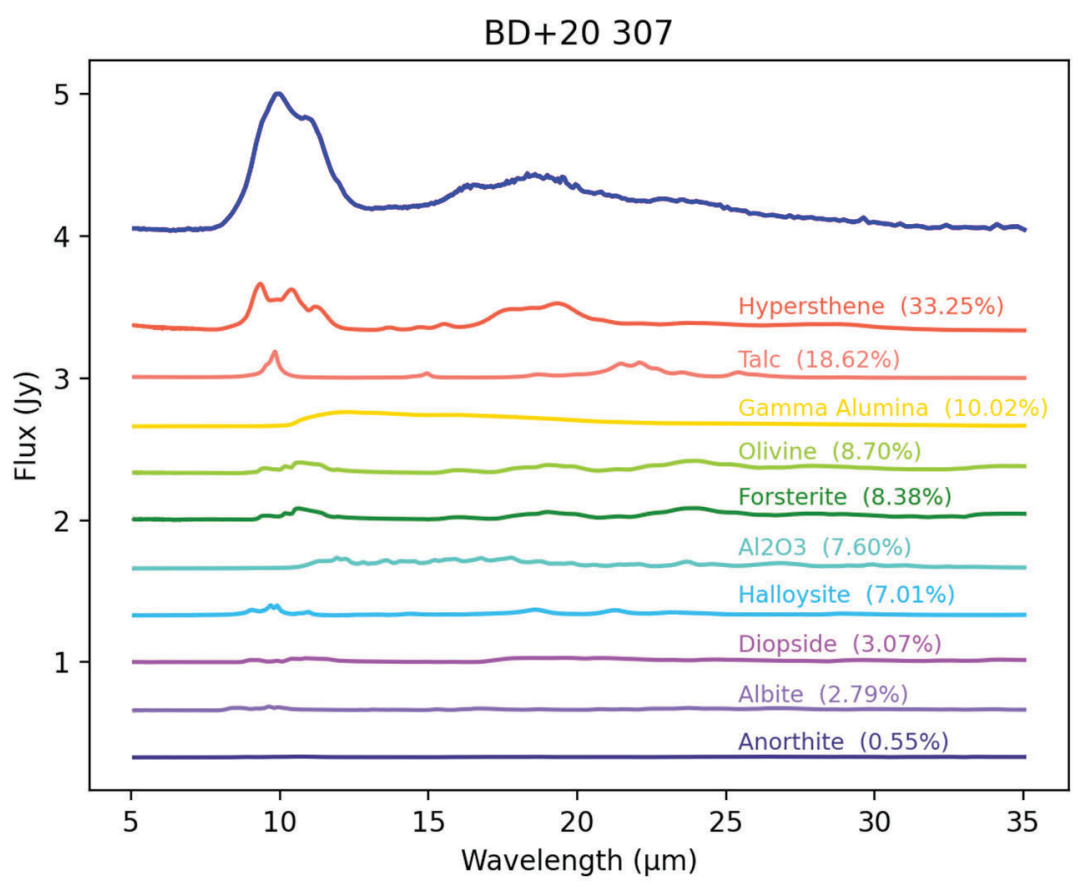

Figure 7. This is a diagram of the Min-CaLM results for the circumstellar disk surrounding BD+20 307 . The recreated spectrum is placed at the top (shown in blue) and is overlayed on top of the observed disk spectrum (shown in red). Note that the recreated spectrum (blue) matches the original spectrum (red) to the extent that the original spectrum cannot be seen at this scale. The mineral spectra placed underneath the circumstellar disk spectrum are ordered based on their relative abundances within the disk. All spectra shown in this diagram have been artificially raised and separated for display purposes. The amplitude of the mineral spectra is scaled by their relative abundances in the debris disk.

\section{DISCUSSION}

The Relation of Calculated Debris Disk Composition to Our Solar System

Of all known debris disks, around 1-2\%, such as the disks analyzed by Min-CaLM, contain a warm infrared excess emission that is created by the collision of planetesimals within the disk. ${ }^{37}$ When Min-CaLM calculates the relative mineral abundance of each debris disk, the abundances represent the composition of the planetesimals that created the dust in the collisions. Therefore, a way to relate the Min-CaLM-calculated debris disk compositions to our solar system is to compare them to the asteroids that are present in our system. One way to do this is to use the Tholen Taxonomy of asteroids.

The Tholen Taxonomy of asteroids categorizes asteroids into different classes based on the albedo and spectral shape of each type of asteroid, and each class of asteroids is found in different regions in the solar system. ${ }^{38}$ An asteroid class provides insight into the composition of the asteroid and its origin within the solar system. ${ }^{39}$ Three of the most abundant asteroid classes found in our solar system are the C, S, and D classes. For comparisons of the composition of the debris disks and asteroid classes, the relative mineral abundances for each debris disk can be found in Table 2, and the group of each mineral in the mineralogical library can be found in Table 1.

The $\mathrm{C}$ asteroid class is believed to be the parent body of carbonaceous chondrite meteorites, meaning that they have a similar composition to chondrites. ${ }^{40} \mathrm{C}$-type asteroids are found in the outer region of the asteroid belt and contain a significant amount of hydrated silicates, also known as phyllosilicates. ${ }^{41}$ Of the debris disks analyzed by Min-CaLM, six of them, HD 23514, HD 105234, HD 15407A, BD+20 307, HD 69830, and HD 172555 have mineral compositions that resemble the composition that is expected for $\mathrm{C}$-type asteroids. This could indicate that $\mathrm{C}$-type asteroids are abundant in these debris disks because the dust in the disks is likely created by the collisions of such asteroids.

S-type asteroids are found primarily in the inner region of the asteroid belt. The S asteroid class is thought to be the parent body to ordinary chondrites, which are the most common form of chondrites. The composition of S-type asteroids varies widely, and so this class is separated into multiple different subtypes. These subtypes are based on the proportion of olivine minerals to various pyroxene mineral groups, such as clinopyroxenes. ${ }^{42}$ Only one of the debris disks analyzed by Min-CaLM is found to have 
a similar composition to S-type asteroids. TYC 9410-532-1 resembles the S(III) subtype, which is characterized by a relative abundance of olivine minerals that is much higher than the abundance of minerals in the pyroxene group. ${ }^{42}$ For example, the debris disk surrounding TYC 9410-532-1 is calculated to be composed of $10.10 \%$ olivine minerals and only $5.40 \%$ pyroxene minerals.

The $\mathrm{D}$ asteroid class can be found past the outer region of the asteroid belt in our solar system. These asteroids are thought to have originated in the Kuiper belt of our solar system. ${ }^{43}$ D-type asteroids are characterized by a lack of phyllosilicates. ${ }^{44}$ Of the debris disks analyzed by Min-CaLM, HD 100546 is found not to contain any phyllosilicate minerals and is therefore most represented by the D-type asteroid type.

The spectrum of the gas-rich protoplanetary disk orbiting HD 100546 is known to be remarkably similar to the spectra of the comets Hale-Bopp and Tempel $1 .{ }^{24}$ Based on the Min-CaLM best-fit result, these objects consists primarily of oxide and silicate group minerals. The similarity in the composition of the debris disk surrounding HD 100546 and the comets could indicate that the environment in which Hale-Bopp and Tempel 1 were created is similar to the current environment of HD $100546 .{ }^{45}$ Because Hale-Bopp and Tempel 1 were likely created in the solar nebula before the creation of planetesimals, this compositional similarity could indicate the presence of forming planetesimals in the circumstellar disk of HD $100546 .{ }^{24}$

\section{Literature Comparison}

The mineral compositions calculated by Min-CaLM for circumstellar disks differ slightly from the compositions reported in other papers. This difference is expected because the system of linear equations displayed in Figure 4 is overdetermined, which indicates that there are many different solutions to the system. The mineral compositions of each debris disk, displayed in Table 2 , are only one possible set of solutions to this degenerate system. This degeneracy is naturally occurring and cannot be removed from consideration. This becomes more apparent when comparing the composition of debris disks to asteroid types. An example of this can be seen with the composition of HD 69830. The composition that Min-CaLM calculated for HD 69830 is most similar to a C-type asteroid, but in Lisse et al. (2006), it is estimated to have a composition more similar to a P or D asteroid. ${ }^{24}$ This difference in asteroid types is a reminder that, because this system is overdetermined, there are many possible solutions for the same system.

Another example of the difference in results can be seen in Lisse et al. (2009). In that paper, the mineral composition of HD 172555 was found to be primarily made of tectosilicates, SiO gas, and olivine minerals. In contrast, Min-CaLM calculated the mineral composition of HD 172555 to contain primarily hornblende, fayalite, and magnetite, which are inosilicates, olivine minerals, and oxide minerals respectively (Table 2 ).

We note that a manual fitting method, such as that applied by Lisse and collaborators, can be beneficial to create traditionally acceptable and appropriate solutions by first considering the minerals that frequently appear in astronomical environments in the fitting procedure. Our method does not take the astrophysical priorities of mineral species into account, and therefore, produces unbiased mathematical solutions. When analyzing a large set of mid-IR disk emission features, such as the ones to be taken with JWST, a quick, unbiased fitting by Min-CaLM followed by detailed manual fitting can be a valuable approach.

\section{Future Improvements}

There are several ways that the Min-CaLM program can be improved in the future. A substantial improvement that can be made to Min-CaLM's mineralogical library is to increase the number of minerals that it contains. This expansion of the mineralogical library would include minerals that are found in debris disks, but whose mid-IR spectrum could not be found when creating the library, such as amorphous refractory species. The increase in the number of minerals could potentially make the Min-CaLMcalculated debris disk compositions more scientifically accurate. Currently, the Min-CaLM library contains forty minerals that were chosen by their occurrence in debris disks, as reported in Lisse et al. 2006, Lisse et al. 2007, and Lisse et al. 2009. Our current spectral library of 40 minerals is likely insufficient to fit a variety of debris disk spectra. Because of the missing mineral spectra, some of the spectral features of the debris disks might be unable to be recreated by Min-CaLM. This effect can be seen most clearly in the spectrum recreation of Hale Bopp (Figure 5). There is a spectral peak around $\sim 24 \mu \mathrm{m}$ that Min-CaLM was not able to reproduce, potentially because it is missing the mineral that creates a peak at that wavelength. However, this discrepancy in peak location could also arise from differences in mineral sample grain shape, porosity, annealing history, and crystallinity, all of which Min-CaLM does not take into consideration. ${ }^{46}$ Note that an increase of the size of Min-CaLM's mineralogical library would result in an increased number of degenerate solutions, as can be seen in Table

4. In this case, the Min-CaLM program would still choose the mineral composition that best fits the debris disk spectrum. 
Another issue is that the mineral spectra included in Min-CaLM's mineralogical library only contains spectra from finely milled laboratory samples. When tiny particles are heated, their thermal spectra contain more prominent peaks than the spectrum that is created by larger particles. This problem can be fixed by expanding the mineralogical library to include different grain sizes for each mineral spectrum. With this improvement, the Min-CaLM program output would also include the grain size that was used for each mineral spectrum found in the debris disk.

Besides improvements on the mineralogical library, a potential future change to Min-CaLM could be to add additional linear regression technique such as Non-Negative Weighted Least Square (NNWLS) regression. This technique would place a weighted probability on each mineral based on how likely that mineral is to be found in a debris disk. Additionally, it could be used to make Min-CaLM place greater importance on certain spectral features when recreating the debris disk spectrum. For instance, it might be more crucial to fit the most prominent spectral peaks of the debris disk than it is to fit the $\sim 40 \mu \mathrm{m}$ tail of the spectrum, which generally contains more noise..$^{47}$ Using NNWLS could provide an additional solution to the overdetermined system of the debris disk spectrum, the relative mineral abundances, and the mineral spectra. A caveat to add the NNWLS linear regression technique is that in certain cases, an emphasis on certain spectral features could bias the data analysis, particularly of outlier data.

Another possible improvement to the Min-CaLM algorithm is to use a regression technique called Non-Negative Least ChiSquare regression (NNLC). This regression technique differs from NNLS by minimizing the (chi-square) uncertainty instead of minimizing the residue difference between the observed spectrum and the recreated spectrum, as NNLS does. ${ }^{48}$ Within the debris disk spectra studied in this paper, the uncertainty values from IRS spectrophotometric flux are the main source of uncertainty. Taking this into consideration would allow the significance of our results to be quantified. While uncertainty calculations are outside the scope of this paper, in future versions of Min-CaLM this could be remedied by using the NNLC regression technique, or a combination of NNLC and NNWLS.

There are several issues regarding the fitting of the mid-IR spectra of debris disks that this paper and the Min-CaLM program does not take into account. First, the mineral spectra in Min-CaLM's mineralogical library contains mostly pure grains with very little impurities. The "dirtiness" of the dust grains affects how efficiently the grains can absorb energy, and therefore are an important feature to consider. Another issue is that only very small grains produce the mineral features that Min-CaLM studies in debris disk spectra. Any minerals that are bound within large dust grains cannot be studied by Min-CaLM because those grains do not produce silicate mineral features in the mid-IR range. Therefore, the mineral compositions of each debris disk, shown in Table 2, are representative of the small grains within the disk, but not necessarily of the large grains. Additionally, the connection between the mineral composition of the dust within the debris disk and the processed material found in asteroids and comets is not entirely straightforward. Small dust grains are blown out of the debris disk by radiation pressure and must be continuously replenished via asteroid or comet collisions for there to be a debris disk. In this paper, we have assumed that small dust grains have a similar composition to the asteroidal material in the debris disk. In nature, dust grains exist with a range of different sizes and a power-law grain size distribution $\left(\mathrm{da} / \mathrm{dN} \sim \mathrm{a}^{\wedge}-3.5\right)$ is commonly used to simulate the size effect. ${ }^{49}$ Smaller grains are superheated due to their low emissivities and different size grains are all emitting at different temperatures. In Min-CaLM, we are effectively fitting observed spectra with a single grain size emissivity data. This limitation may be the cause of slightly different fitting results obtained in our method versus other literature results. A future improvement is expected to address this grain size issue.

The Min-CaLM program is still in the early stages of development. As previously stated, Min-CaLM does not consider the effects that grain size, different particle temperatures, or porosities in the grains have on the produced spectrum. It also does not consider how the different laboratories produced the input emissivity mineral spectra that are used in Min-CaLM's mineralogical library and what effect that has on the Min-CaLM output. Overall, this paper serves as a demonstration of the potential that this program has in one day becoming a useful tool in the astronomy community. The findings in this paper should be taken with the understanding that the Min-CaLM program is still in development and that future generations of the program will incorporate the improvements discussed in this section.

The Min-CaLM program produces an unbiased mineral composition for debris disks that are capable of undergoing mineralogical compositional analysis. With new debris disk spectral data from the James Webb Space Telescope, the Min-CaLM program and the linear regression method behind it could be refined further to provide a useful platform for future analyses.

Min-CaLM has been published in the Astrophysics Source Code Library (ASCL) under the bibcode 2020ascl.soft01001K. Both the Min-CaLM program and mineralogical library can be downloaded from a GitHub repository that is linked in the ASCL page. 


\section{ACKNOWLEDGEMENTS}

The authors thank the University of Georgia, the UGA Department of Physics \& Astronomy, and the support from the UGA Center for Undergraduate Research Opportunities. The authors thank the referees (two anonymous ones and Dr. Lisse) for their helpful constructive inputs.

\section{REFERENCES}

1. Andrews, S., Wilner, D., Hughes, A., Qi, C., Dullemond, C. (2009) "PROTOPLANETARY DISK STRUCTURES IN OPHIUCHUS Protoplanetary Disk Structures in Ophiuchus", The Astrophysical Journal, 700(2), 1502-1523. doi:10.1088/0004$637 X / 700 / 2 / 1502$

2. Henning, T. (2010). Cosmic Silicates. Annual Review of Astronomy and Astrophysics, 48(1), pp.21-46. bttps:// doi.org/10.1146/ annurev-astro-081309-130815

3. Takasawa, S., Nakamura, A., Kadono, T., Arakawa, M., Dohi, K., Ohno, S., Seto, Y., Maeda, M., Shigemori, K., Hironaka, Y., Sakaiya, T., Fujioka, S., Sano, T., Otani, K., Watari, T., Sangen, K., Setoh, M., Machii, N., Takeuchi, T. (2011) "Silicate Dust Size Distribution from Hypervelocity Collisions: Implications for Dust Production in Debris Disks", The Astrophysical Journal, 733(2), L39. https:// doi.org/10.1088/2041-8205/733/2/L39

4. Su, K., Rieke, G., Stansberry, J., Bryden, G., Stapelfeldt, K., Trilling, D., Muzerolle, J., Beichman, C., Moro-Martin, A., Hines, D., Werner, M. (2006) "Debris Disk Evolution around A Stars", The Astrophysical Journal, 653(1), 675-689. bttps:// doi.org/10.1086/508649

5. Hughes, A., Duchêne, G. and Matthews, B. (2018). Debris Disks: Structure, Composition, and Variability. Annual Review of Astronomy and Astrophysics, 56(1), pp.541-591. https:// doi.org/10.1146/ annurev-astro-081817-052035

6. Plavchan, P., Jura, M. and Lipscy, S. (2005). "Where Are the M Dwarf Disks Older Than 10 Million Years?”. The Astrophysical Journal, 631(2), pp.1161-1169. https:// doi.org/10.1086/432568

7. Klahr, H., Lin, D. (2001) "Dust Distribution in Gas Disks: A Model for the Ring around HR 4796A", The Astrophysical Journal, 554(2), 1095-1109. bttps:// doi.org/10.1086/321419

8. Wood, K., Lada, C., Bjorkman, J., Kenyon, S., Whitney, B., Wolff, M. (2002) "Infrared Signatures of Protoplanetary Disk Evolution", The Astrophysical Journal, 567(2), 1183-1191. bttps:// doi.org/10.1086/338662

9. Wolf, S., Hillenbrand, L. (2003) "Model Spectral Energy Distributions of Circumstellar Debris Disks. I. Analytic Disk Density Distributions", The Astrophysical Journal, 596(1), 603-620. https:// doi.org/10.1086/377638

10. Clark, R. (1999) Remote Sensing for the Earth Sciences: Manual of Remote Sensing, 3rd ed, John Wiley \& Sons, Inc.

11. Morlok, A., Koike, C., Tomioka, N., Mann, I., Tomeoka, K. (2010) "Mid-infrared spectra of the shocked Murchison CM chondrite: Comparison with astronomical observations of dust in debris disks", Icarus, 207(1), 45-53. https:// doi.org/10.1016/j.icarus.2009.11.018

12. Scott, E., Krot, A. (2005) "Thermal Processing of Silicate Dust in the Solar Nebula: Clues from Primitive Chondrite Matrices", The Astrophysical Journal, 623(1), 571-578. bttps:// doi.org/10.1086/428606

13. Hewins, R., Zanda, B., Bendersky, C. (2012) "Evaporation and recondensation of sodium in Semarkona Type II chondrules", Geochimica et Cosmochimica Acta, 78, 1-17. bttps: / / doi.org/10.1016/j.gca.2011.11.027

14. Moro-Martín, A. (2007) "On the solar system—debris disk connection", Proceedings of the International Astronomical Union, 3(S249), 347-354. bttps:// doi.org/10.1017/S1743921308016803

15. Meyer, M.R., Hillenbrand, L.A., Backman, D., Beckwith, S., Bouwman, J., Brooke, T., Carpenter, J., Cohen, M., Cortes, S., Crockett, N., Gorti, U., Henning, T., Hines, D., Hollenbach, D., Kim, J.S., Lunine, J., Malhotra, R., Mamajek, E., Metchev, S., Moro-Martin, A., Morris, P., Najita, J., Padgett, D., Pascucci, I., Rodmann, J., Schlingman, W., Silverstone, M., Soderblom, D., Stauffer, J., Stobie, E., Strom, S., Watson, D., Weidenschilling, S., Wolf, S., Young, E. (2006) “The Formation and Evolution of Planetary Systems: Placing Our Solar System in Context with Spitzer," Publications of the Astronomical Society of the Pacific, 118(850), 1690-1710.

16. Eiroa, C., Marshall, J.P., Mora, A., Montesinos, B., Absil, O., Augereau, J.C., Bayo, A., Bryden, G., Danchi, W., del Burgo, C., Ertel, S., Fridlund, M., Heras, A.M., Krivov, A.V., Launhardt, R., Liseau, R., Löhne, T., Maldonado, J., Pilbratt, G.L., Roberge, A., Rodmann, J., Sanz-Forcada, J., Solano, E., Stapelfeldt, K., Thébault, P., Wolf, S., Ardila, D., Arévalo, M., Beichmann, C., Faramaz, V., González-García, B.M., Gutiérrez, R., Lebreton, J., Martínez-Arnáiz, R., Meeus, G., Montez, D., Olofsson, G., Su, K.Y.L., White, G.J., Barrado, D., Fukagawa, M., Grün, E., Kamp, I., Lorente, R., Morbidelli, A., Müller, S., Mutschke, H., Nakagawa, T., Ribas, I., Walker, H. (2013) “DUst around NEarby Stars. The survey observational results," Astronomy \& Astrophysics, 555. bttps:// doi.org/10.1051/0004-6361/201321050.

17. Cotten, T., Song, I. (2016) "A Comprehensive Census of Nearby Infrared Excess Stars", The Astrophysical Journal Supplement Series, 225(1), 15. bttps:// doi.org/10.3847/0067-0049/225/1/15

18. Mumma, M. (1997) "Organic Volatiles in Comets: Their Relation to Interstellar Ices and Solar Nebula Material", From Stardust to Planetesimals, $122,369$.

19. Huebner, W. (2002) "Composition of Comets: Observations and Models", Earth, Moon, and Planets, 89(1-4), 179-195. https:// doi.org/10.1023/A:10215068 
20. Wooden, D., Harker, D., Woodward, C. (1999) "Crystalline Silicates, Comets, and Protoplanetary Disk Evolution", Thermal Emission Spectroscopy and Analysis of Dust, Disks, and Regoliths, Proceedings of a meeting held at at The Lunar and Planetary Institute, vol. 196(Astronomical Society of the Pacific Conference Series), 99-108.

21. Harker, D., Woodward, C., Wooden, D. (2005) "The Dust Grains from 9P/Tempel 1 Before and After the Encounter with Deep Impact", Science, 310(5746), 278-280.

22. A'Hearn, M., Belton, M., Delamere, W., Thomas, P., Kissel, J., Klaasen, K., McFadden, L.,Meech,M.J, Melosh, H.J., Shultz, P.H., Sunshine, J.M., Veverka, J., Yeomans, D.K., Baca, M.W., Busko, I., Crokett, C.J., Collins, S.M., Desnoyer, M., Eberhardy, C.A., Ernst, C.M., Farnham, T.L., Feaga, L., Groussin, O., Hampton, D., Ipatov, S.I., Li, J.Y., Linder, D., Lisse, C.M., Mastrodemos, N., Owen Jr., W.M., Richardson, J.E., Wellmitz, White, R.L. (2005) "Deep Impact: Excavating Comet Tempel 1", Science, 310(5746), 258-264. https:// doi.org/10.1126/ science.1118923

23. Lisse, C., VanCleve, J., Adams, A., A'Hearn, M., Fernández, Y., Farnham, T., Armus, L., Grillmair, C., Ingalls, J., Belton, M., Groussin, O., McFadden, L., Meech, K., Schultz, P., Clark, B., Feaga, L., Sunshine, J. (2006) "Spitzer Spectral Observations of the Deep Impact Ejecta", Science, 313(5787), 635-640. https:// doi.org 10.1126/ science.1124694

24. Lisse, C., Beichman, C., Bryden, G., Wyatt, M. (2007) "On the Nature of the Dust in the Debris Disk around HD 69830", The Astrophysical Journal, 658(1), 584-592. https:/ / doi.org/10.1086/511001

25. Backman, D., Marengo, M., Stapelfeldt, K., Su, K., Wilner, D., Dowell, C., Watson, D., Stansberry, J., Rieke, G., Megeath, T., Fazio, G., Werner, M. (2008) " Epsilon Eridani's Planetary Debris Disk: Structure and Dynamics Based on Spitzer and Caltech Submillimeter Observatory Observations", The Astrophysical Journal, 690(2), 1522-1538. https://doi.org/10.1088/0004$637 X / 690 / 2 / 1522$

26. Wu, X., Roby, T., Ly, L. (2010) "Spitzer Heritage Archive”, Observatory Operations: Strategies, Processes, and Systems. https:// doi.org/ 10.1117/12.857728.

27. Brandl, B., Bernard-Salas, J., Spoon, H., Devost, D., Sloan, G., Guilles, S., Wu, Y., Houck, J., Weedman, D., Armus, L., Appleton, P., Soifer, B., Charmandaris, V., Hao, L., Higdon, J., Herter, T. (2006) "The Mid-Infrared Properties of Starburst Galaxies from Spitzer-IRS Spectroscopy", The Astrophysical Journal, 653(2), 1129-1144.

28. Wu, Y., Charmandaris, V., Hao, L., Brandl, B., Bernard-Salas, J., Spoon, H., Houck, J. (2006) "Mid-Infrared Properties of Low-Metallicity Blue Compact Dwarf Galaxies from the Spitzer Infrared Spectrograph", The Astrophysical Journal, 639(1), 157172. https:// doi.org/10.1086/499226.

29. Hernán-Caballero, A., Spoon, H., Lebouteiller, V., Rupke, D. and Barry, D. (2015). The infrared database of extragalactic observables from Spitzer - I. The redshift catalogue. Monthly Notices of the Royal Astronomical Society, 455(2), pp.1796-1806. bttps: / / doi.org/10.1093/mnras/stv2464

30. Combined Atlas Of Sources With Spitzer IRS Spectra: Search [online] (2019) [online], cassis.sirtf.com.

31. Lebouteiller, V., Barry, D., Goes, C., Sloan, G., Spoon, H., Weedman, D., Bernard-Salas, J. and Houck, J., 2015. "CASSIS: The Cornell Atlas of Spitzer / Infrared Spectrograph Sources. II. High-resolution Observations”. The Astrophysical Journal Supplement Series, 218(2), p.21. bttps:// doi.org/10.1088/0067-0049/218/2/21.

32. Currie, T., Lisse, C., Sicilia-Aguilar, A., Rieke, G., Su, K. (2011) " Spitzer Infrared Spectrograph Spectroscopy of the 10 Myr Old EF Cha Debris Disk: Evidence for Phyllosilicate-Rich Dust in the Terrestrial Zone", The Astrophysical Journal, $734(2), 115$. bttps://doi.org/10.1088/0004-637X/734/2/115

33. Zyskind, G. (1967) "On Canonical Forms, Non-Negative Covariance Matrices and Best and Simple Least Squares Linear Estimators in Linear Models", The Annals of Mathematical Statistics, 38(4), 1092-1109. bttps:/ / doi.org/ 10.1214/ aoms/1177698779

34. Williams, G. (1990) "Overdetermined Systems of Linear Equations", The American Mathematical Monthly, $97(6), 511$. bttps:// doi.org/10.1080/00029890.1990.11995638

35. Andrae, R., Schulze-Hartung, T., Melchior, P. (2020) Dos and Don'ts of Reduced Chi-Squared [online], Arxiv.org, available: https://arxiv.org/pdf/1012.3754.pdf [accessed 25 Oct 2020].

36. Morlok, A., Lisse, C., Mason, A., Bullock, E., Grady, M. (2014) "Mid-infrared spectroscopy of components in chondrites: Search for processed materials in young Solar Systems and comets", Icarus, 231, 338-355. https:// doi.org/10.1016/j.icarus.2013.12.018.

37. Morlok, A., Mason, A., Anand, M., Lisse, C., Bullock, E., Grady, M. (2014) "Dust from collisions: A way to probe the composition of exo-planets?", Icarus, 239, 1-14. https:// doi.org/10.1016/j.icarus.2014.05.024.

38. Lodders, K., Fegley, B. (1998) The Planetary Scientist's Companion, Oxford University Press: New York.

39. Britt, D., Tholen, D., Bell, J., Pieters, C. (1992) "Comparison of asteroid and meteorite spectra: Classification by principal component analysis", Icarus, 99(1), 153-166. https:/ / doi.org/10.1016/0019-1035(92)90179-B

40. Busarev, V. (2012) "A hypothesis on the origin of C-type asteroids and carbonaceous chondrites", Asteroids, Comets, Meteors, 1667.

41. Richard, N. (2002) "The Cambridge encyclopedia of meteorites", Choice Reviews Online, 40(02), 40-0661-40-0661.

42. Gaffey, M., Burbine, T., Binzel, R. (1993) "Asteroid spectroscopy: Progress and perspectives", Meteoritics, 28(2), $161-187$. https://doi.org/10.1111/j.1945-5100.1993.tb00755.x 
43. McKinnon, W. (2008) "On The Possibility Of Large KBOs Being Injected Into The Outer Asteroid Belt", American Astronomical Society, Vol. 40, 464.

44. Cruikshank, D., Dalle Ore, C., Roush, T., Geballe, T., Owen, T., de Bergh, C., Cash, M., Hartmann, W. (2001) "Constraints on the Composition of Trojan Asteroid 624 Hektor", Icarus, 153(2), 348-360.

45. Bouwman, J., de Koter, A., Dominik, C., Waters, L. (2003) "The origin of crystalline silicates in the Herbig Be star HD 100546 and in comet Hale-Bopp", Astronomy \& Astrophysics, 401(2), 577-592. https:/ / doi.org/ 10.1051/0004-6361:20030043

46. Olofsson, J., Juhász, A., Henning, T., Mutschke, H., Tamanai, A., Moór, A., Ábrahám, P. (2012) "Transient dust in warm debris disks", Astronomy \& Astrophysics, 542, A90. bttps:// doi.org/10.1051/0004-6361/201118735.

47. Teplitz, H., Desai, V., Armus, L., Chary, R., Marshall, J., Colbert, J., Frayer, D., Pope, A., Blain, A., Spoon, H., Charmandaris, V., Scott, D. (2007) "Measuring PAH Emission in Ultradeep Spitzer IRS Spectroscopy of High-Redshift IR-Luminous Galaxies", The Astrophysical Journal, 659(2), 941-949. https:/ / doi.org/10.1086/512802

48. Désesquelles, P., Ha, T.M.H., Korichi, A., Blanc, F.L., Petrache, C.M. (2009) "NNLC: non-negative least chi-square minimization and application to HPGe detectors," Journal of Physics G: Nuclear and Particle Physics, 36(3), 037001. https://doi.org/10.1088/0954-3899/36/3/037001.

49. MacGregor, Meredith A., et al. "Constraints on Planetesimal Collision Models in Debris Disks." The Astrophysical Journal, vol. 823, no. 2, 2016, p. 79., doi:10.3847/0004-637x/823/2/79. bttp://dx.doi.org/10.3847/0004-637X/823/2/79

\section{ABOUT STUDENT AUTHORS}

Yung Kipreos graduated from the University of Georgia in May 2020 with a major in Astrophysics and a minor in Computer Science. She wants to attend graduate school to become an exoplanetary scientist.

\section{PRESS SUMMARY}

A circumstellar disk surrounding a star is composed of gas, dust, and rocky objects in orbit around the star. Around infant stars, this disk can act as a source of material to form planetesimals, which can then accrete more material and form into planets. Studying the mineral composition of these disks can provide insight into the processes that created our solar system. In this paper, we introduce a new unbiased mineral compositional analysis technique, Min-CaLM, and apply it to eight circumstellar disks around the stars: HD 23514, HD 105234, HD 15407A, BD+20 307, HD 69830, and HD 172555. The Min-CaLM python package is open-access and is available to download from https://github.com/yungkipreos/Min-CaLM. 